Mathematical Research Letters 9, 1-15 (2002)

\title{
THE CASTELNUOVO-MUMFORD REGULARITY OF AN INTEGRAL VARIETY OF A VECTOR FIELD ON PROJECTIVE SPACE
}

\author{
EduARdo Esteves
}

\begin{abstract}
The Castelnuovo-Mumford regularity $r$ of a variety $V \subseteq \mathbf{P}_{\mathbf{C}}^{n}$ is an upper bound for the degrees of the hypersurfaces necessary to cut out $V$. In this note we give a bound for $r$ when $V$ is left invariant by a vector field on $\mathbf{P}_{\mathbf{C}}^{n}$. More precisely, assume $V$ is arithmetically Cohen-Macaulay, for instance, a complete intersection. Assume as well that $V$ projects to a normal-crossings hypersurface, which is the case when $V$ is a curve with at most ordinary nodes. Then we show that $r \leq m+s+1$, where $s$ is the dimension of $V$ and $m$ is the degree of the vector field. Our method consists of using first central projections to reduce the problem to when $V$ is a hypersurface, and then using bounds given by Brunella and Mendes.
\end{abstract}

\section{Introduction}

Let $k$ be an algebraically closed field of characteristic $p \geq 0$, and $\mathbf{P}_{k}^{n}$ the $n$ dimensional projective space over $k$. Let $X$ be an algebraic vector field on $\mathbf{P}_{k}^{n}$. A subscheme $V \subseteq \mathbf{P}_{k}^{n}$ is said to be left invariant by $X$, or an integral subscheme of $X$, if $X$ restricts to a vector field on $V$. For $k=\mathbf{C}$, the vector field $X$ induces a holomorphic flow away from its singular points. One might ask whether there are complex, compact subvarieties of $\mathbf{P}_{\mathbf{C}}^{n}$ where the flow stays confined. These subvarieties are algebraic by Chow's theorem, and are left invariant by $X$.

For $k=\mathbf{C}$ and $n=2, \mathrm{H}$. Poincaré $[\mathrm{P}]$ considered the following question: Can we give a bound for the degrees of plane curves left invariant by $X$ ? If a bound were given, then we could restrict the search for such curves over a finite-dimensional vector space, that of polynomials of degree at most the given bound. In addition, it would be interesting if the bound depended only on the unique numerical invariant of $X$, its degree. For general $k$ and $n$, the degree of $X$ is the number of points on a general hyperplane $H \subseteq \mathbf{P}_{k}^{n}$ at which the direction given by $X$ lies in $H$. Let $m$ denote the degree of $X$.

It's impossible to find the bound asked by Poincaré for $k=\mathbf{C}$ and $n=2$ only in terms of $m$; see Remark 9. However, given a reduced curve $C \subseteq \mathbf{P}_{\mathbf{C}}^{2}$ of degree $d$ left invariant by $X$, bounds for $d$ in terms of $m$ were found under

Received November 2, 2000.

Supported by PRONEX, Convênio 41/96/0883/00, CNPq, Proc. 300004/95-8, and FAPERJ, Proc. E-26/170.418/2000-APQ1. 
certain conditions on $X$ or $C$. For instance, if the singularities of $X$ are nondiacritical, then M. Carnicer showed in [C] that $d \leq m+2$. If $C$ has at most ordinary nodes for singularities, D. Cerveau and A. Lins Neto showed in [CL] that $d \leq m+2$ as well, with equality only if $C$ is reducible. If $C$ has worse singularities or $X$ is diacritical, the above inequality does not hold. For these cases, weaker inequalities were given by A. Campillo and Carnicer in [CC].

For $k=\mathbf{C}$ but general $n$, M. Soares showed that the inequality $d \leq m+1$ holds for the degree $d$ of a smooth hypersurface left invariant by $X$; see [So1]. Recently, M. Brunella and L.G. Mendes showed that a hypersurface of $\mathbf{P}_{\mathbf{C}}^{n}$ left invariant by $X$ has degree at most $m+n$ if it has at most normal-crossings singularities; see $[\mathrm{BM}]$. (More generally, they bounded the degrees of solutions to Pfaff equations on complex, projective manifolds $M$ with $\operatorname{Pic}(M) \cong \mathbb{Z}$.)

Further results are obtained for complete intersections. If $C \subseteq \mathbf{P}_{\mathbf{C}}^{n}$ is a complete intersection of hypersurfaces of degrees $d_{1}, \ldots, d_{n-1}$, and is left invariant by $X$, a quite simple inequality was obtained by Soares in [So2]:

$$
d_{1}+\cdots+d_{n-1} \leq m+n-1 \text { if } C \text { is smooth. }
$$

If $C$ has at most ordinary nodes for singularities, Campillo, Carnicer and J. García de la Fuente showed in $[\mathrm{CCF}]$ that $\sum d_{i} \leq m+n$. They gave also inequalities for when $C$ has worse singularities.

In the present note we give a direct algebraic proof of Soares' result, which is essentially an observation by O. Zariski; see Theorem 8. Furthermore, the proof yields a characterization of all vector fields that leave invariant a given smooth hypersurface; see Theorem 7.

Most importantly, we treat the case $n \geq 3$. Following up on Poincaré's original intention, we want to restrict to a finite-dimensional space the search for a subscheme $V \subseteq \mathbf{P}_{k}^{n}$ left invariant by $X$. This is more directly achieved not by bounding the degree of a possible $V$, but by bounding the degrees of the polynomials necessary to cut out $V$. These degrees are bounded by the Castelnuovo-Mumford regularity of $V$, defined below.

Given a subscheme $V \subseteq \mathbf{P}_{k}^{n}$, let $I$ be its saturated homogeneous ideal, and

$$
\cdots \rightarrow F_{i} \rightarrow \cdots \rightarrow F_{0} \rightarrow I \rightarrow 0
$$

the minimal graded free resolution of $I$. For each non-negative integer $i$, let $b_{i}$ be the maximum of the degrees of the generators of $F_{i}$. The Castelnuovo-Mumford regularity, or simply regularity, of $V$ is the integer $r$ given as

$$
r=\max \left(b_{i}-i \mid i=0,1, \ldots\right)
$$

The above definition of regularity is of a rather arithmetical nature. However, if $V$ is arithmetically Cohen-Macaulay, by which we mean that its ring of homogeneous coordinates is Cohen-Macaulay, the regularity acquires a more geometric meaning. More precisely, cut out $V$ by as many general hyperplanes as its dimension to obtain a set $\Gamma$ of points. Then the regularity of $V$ is equal to that of $\Gamma$, and the regularity of $\Gamma$ is the smallest integer $r$ such that for each $P \in \Gamma$ 
there is a hypersurface of degree $r-1$ passing through all the points of $\Gamma$ but $P$. So, points disposed in special position tend to have higher regularity.

Complete intersections are arithmetically Cohen-Macaulay. The regularity of a hypersurface is its degree. Hence, the next theorem is a generalization of the inequality found by Cerveau and Lins Neto on the complex plane.

Theorem 1. Let $C \subseteq \mathbf{P}_{k}^{n}$ be a reduced, arithmetically Cohen-Macaulay curve with at most ordinary nodes for singularities, degree $d$ and Castelnuovo-Mumford regularity $r$. If $C$ is left invariant by a vector field $X$ of degree $m$ on $\mathbf{P}_{k}^{n}$, and contains only finitely many singularities of $X$, then $r \leq m+2$, with equality only if $C$ is reducible or $p \mid d$.

A generalization of Theorem 1 is stated as Theorem 18, and follows from the main result of this note, Theorem 17. The assumption that $C \subseteq \mathbf{P}_{k}^{n}$ be arithmetically Cohen-Macaulay is not so restrictive as it may seem. If $C$ is smooth, it's the same as assuming that $C$ be projectively normal. If $n=3$, it follows from the Hilbert-Burch Theorem that the set of arithmetically CohenMacaulay curves in $\mathbf{P}_{k}^{3}$ is open in the Hilbert scheme; see [Se].

Corollary 2. Let $C \subseteq \mathbf{P}_{k}^{n}$ be a reduced curve with at most ordinary nodes for singularities. Assume $C$ is the complete intersection of hypersurfaces of degrees $d_{1}, d_{2}, \ldots, d_{n-1}$. If $C$ is left invariant by a vector field $X$ of degree $m$ on $\mathbf{P}_{k}^{n}$, and contains only finitely many singularities of $X$, then

$$
d_{1}+\cdots+d_{n-1} \leq m+n,
$$

with equality only if $C$ is reducible or $p \mid\left(d_{1} \cdots d_{n-1}\right)$.

As complete intersections are arithmetically Cohen-Macaulay, to get the above inequality it is enough to observe that the regularity of $C$ is $d_{1}+\cdots+$ $d_{n-1}-n+2$, what follows from considering the Koszul resolution of the homogeneous ideal of $C$. For $C$ irreducible, our inequality is sharper than that given in $[\mathrm{CCF}]$. (The inequality given in $[\mathrm{CCF}]$ is recovered more simply by our Proposition 12.)

Our technique is quite simple, and applicable in greater generality. Given a reduced, closed subscheme $V \subseteq \mathbf{P}_{k}^{n}$ of dimension $s$, we can project it to a hypersurface $\bar{V} \subseteq \mathbf{P}_{k}^{s+1}$. If the center of projection is general enough, then $\bar{V}$ will have the same degree of $V$ and singularities that are not much worse. (If $V$ is a curve with at most ordinary nodes for singularities, so is $\bar{V}$.) Now, if $X$ is a vector field on $\mathbf{P}_{k}^{n}$ leaving $V$ invariant, can we find a vector field $\bar{X}$ on $\mathbf{P}_{k}^{s+1}$ of degree not much higher than that of $X$ leaving $\bar{V}$ invariant? If so, we may use the bounds known for hypersurfaces. Well, the answer to our question is yes, if $V$ is arithmetically Cohen-Macaulay. As our Theorem 17 shows, the difference between the degrees of $\bar{X}$ and $X$ need not be higher than $d-r$, where $d$ is the degree of $V$ and $r$ its regularity. To prove Theorem 17 we use elimination in a quite elementary way.

Theorem 17 reduces our problem of bounding regularity to that of bounding the degree of a hypersurface. For curves, we can use the result by Cerveau 
and Lins Neto. This application of Theorem 17 is our Theorem 18, from which Theorem 1 above follows.

For curves which are not arithmetically Cohen-Macaulay, the next theorem can be applied:

Theorem 3. Let $C \subseteq \mathbf{P}_{k}^{n}$ be a reduced curve with degree $d$ and at most ordinary nodes for singularities. Assume $C \subseteq Z \cap H$, where $Z$ is an arithmetically CohenMacaulay surface of degree e, Castelnuovo-Mumford regularity $r$, and $H$ is a hypersurface of degree $f$ containing no irreducible component and just finitely many singularities of $Z$. If $C$ is left invariant by a vector field $X$ of degree $m$ on $\mathbf{P}_{k}^{n}$, and contains only finitely many singularities of $X$, then

$$
d \leq m+f(e-1)-r+3,
$$

with equality only if $C$ is reducible or $p \mid d$.

The above theorem follows as well from our Theorem 18. If $C \subseteq \mathbf{P}_{k}^{n}$ is a curve with at most ordinary nodes for singularities, it follows from the Bertini theorems of $[\mathrm{AK}]$ that $C$ is contained in a smooth, complete intersection surface $Z \subseteq \mathbf{P}_{k}^{n}$. So, the above theorem is always applicable.

Using the result by Brunella and Mendes, we can generalize Theorem 1.

Theorem 1*. Let $V \subseteq \mathbf{P}_{\mathbf{C}}^{n}$ be a reduced, arithmetically Cohen-Macaulay subscheme of dimension $s$ and Castelnuovo-Mumford regularity $r$. Assume $V$ projects from a general center to a hypersurface with at most normal-crossings singularities. Let $X$ be a vector field of degree $m$ on $\mathbf{P}_{\mathbf{C}}^{n}$ leaving $V$ invariant. Assume each irreducible component of $V$ contains a non-singular point of $X$. Then $r \leq m+s+1$.

Even when the subscheme $V \subseteq \mathbf{P}_{\mathbf{C}}^{n}$ is smooth, even when the center of projection is general, $V$ may project to a hypersurface with worse singularities than normal-crossings.

This note relies heavily on a few results of Commutative Algebra. For the benefit of the non-specialist, I tried to give precise references to these results as much as possible. I'm grateful to S. Kleiman, L.G. Mendes, J.V. Pereira, A. Simis, M. Soares and B. Ulrich for many helpful discussions. I thank also A. Garcia for working out with me Remark 14.

\section{Vector fields}

4. Vector fields. Let $V$ be a scheme over $k$ and $\mathcal{L}$ an invertible sheaf on $V$. A vector field on $V$ with coefficients in $\mathcal{L}$ is a map of $\mathcal{O}_{V}$-modules $X: \Omega_{V}^{1} \rightarrow \mathcal{L}$.

Let $m$ be a non-negative integer. Using the Euler sequence,

$$
0 \rightarrow \Omega_{\mathbf{P}_{k}^{n}}^{1} \rightarrow \mathcal{O}_{\mathbf{P}_{k}^{n}}(-1)^{n+1} \rightarrow \mathcal{O}_{\mathbf{P}_{k}^{n}} \rightarrow 0,
$$

we see that a vector field $X: \Omega_{\mathbf{P}_{k}^{n}}^{1} \rightarrow \mathcal{O}_{\mathbf{P}_{k}^{n}}(m-1)$ is induced by a (homogeneous) vector field,

$$
G_{0} \partial_{0}+\cdots+G_{n} \partial_{n},
$$


on $\mathbf{A}_{k}^{n+1}$, where $G_{0}, \ldots, G_{n} \in k\left[t_{0}, \ldots, t_{n}\right]$ are homogeneous of degree $m$, and $\partial_{0}, \ldots, \partial_{n}$ are the standard derivations with respect to $t_{0}, \ldots, t_{n}$. We say that $X$ is induced by $\sum G_{i} \partial_{i}$. Moreover, any two homogeneous vector fields on $\mathbf{A}_{k}^{n+1}$ determine the same vector field on $\mathbf{P}_{k}^{n}$ if and only if they differ by a multiple of the radial (or Euler) vector field, which is induced by $\sum t_{i} \partial_{i}$. By convention, $X$ has degree $m$.

The vector field $X$ induced by $\sum G_{i} \partial_{i}$ may be viewed as the rational map sending a point $\left(t_{0}: \cdots: t_{n}\right) \in \mathbf{P}_{k}^{n}$ to $\left(G_{0}\left(t_{0}, \ldots, t_{n}\right): \cdots: G_{n}\left(t_{0}, \ldots, t_{n}\right)\right)$. The line passing through these two points is called the direction given by $X$ at $\left(t_{0}: \cdots: t_{n}\right)$. The rational map and the line are defined for $\left(t_{0}: \cdots: t_{n}\right)$ not on the closed subscheme $V \subseteq \mathbf{P}_{k}^{n}$ cut out by the maximal minors of the matrix

$$
\left[\begin{array}{ccc}
t_{0} & \ldots & t_{n} \\
G_{0} & \ldots & G_{n}
\end{array}\right] .
$$

A point $P \in V$ is called a singularity of $X$, or singular for $X$.

5. Integral subschemes. Given a scheme $V$ over $k$ and a vector field $X: \Omega_{V}^{1} \rightarrow \mathcal{L}$, a subscheme $W \subseteq V$ is said to be left invariant by $X$, or an integral subscheme of $X$, if $X$ restricts to a vector field on $W$. More precisely, $W$ is left invariant by $X$ if there is a vector field $Y:\left.\Omega_{W}^{1} \rightarrow \mathcal{L}\right|_{W}$ making the following diagram commute:

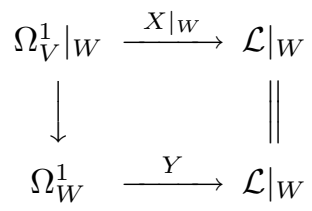

where the left vertical map is the natural restriction. If (2) commutes, we say that $X$ restricts to $Y$ or $Y$ lifts to $X$.

Algebraically, if $X$ is the vector field on $\mathbf{P}_{k}^{n}$ induced by $\sum G_{i} \partial_{i}$, and $V \subseteq$ $\mathbf{P}_{k}^{n}$ is the closed subscheme cut out by the saturated, homogeneous ideal $I \subseteq$ $k\left[t_{0}, \ldots, t_{n}\right]$, then $X$ leaves $V$ invariant if and only if $\sum G_{i} \partial_{i} F \in I$ for each $F \in I$. In fact, if $I$ is any homogeneous ideal cutting out $V$, then $\sum G_{i} \partial_{i} F \in I$ for each $F \in I$ only if $V$ is left invariant by $X$. The converse does not hold in general, if $I$ is not saturated. For instance, the variety cut out by $t_{0}$ is left invariant by the vector field induced by $\partial_{1}$, though $\partial_{1}\left(t_{0} t_{1}\right)$ is not in the ideal generated by $t_{0}^{2}, t_{0} t_{1}, \ldots, t_{0} t_{n}$.

Let $X$ be a vector field on $\mathbf{P}_{k}^{n}$ of degree $m$. If $X \neq 0$, we can give a more geometric meaning to the degree. Indeed, let $H \subseteq \mathbf{P}_{k}^{n}$ be a hyperplane not left invariant by $X$. The points of $H$ at which the direction given by $X$ lies in $H$ are those in the degeneracy scheme $Z$ of the natural map $\left.\Omega_{\mathbf{P}_{k}{ }^{n}}\right|_{H} \rightarrow \Omega_{H}^{1} \oplus \mathcal{O}_{H}(m-1)$ induced by $X$. Now, from the Euler sequences of $\mathbf{P}_{k}^{n}$ and $H$ we get that the determinants of $\Omega_{\mathbf{P}_{k}^{n}}^{1}$ and $\Omega_{H}^{1}$ are $\mathcal{O}_{\mathbf{P}_{k}^{n}}(-n-1)$ and $\mathcal{O}_{H}(-n)$. So, $Z$ is the zero scheme of a section of $\mathcal{O}_{H}(m)$, whence has degree $m$. 


\section{Hypersurfaces and curves}

6. Hypersurfaces. Let $V \subseteq \mathbf{P}_{k}^{n}$ be the hypersurface cut out by a homogeneous $F \in k\left[t_{0}, \ldots, t_{n}\right]$. Then the vector field $X$ on $\mathbf{P}_{k}^{n}$ induced by $\sum G_{i} \partial_{i}$ leaves $V$ invariant if and only if

$$
G_{0} \partial_{0} F+\cdots+G_{n} \partial_{n} F=P F
$$

for a certain $P \in k\left[t_{0}, \ldots, t_{n}\right]$. We can easily construct vector fields that leave $V$ invariant, namely those induced by

$$
\sum_{i<j} P_{i, j}\left(\partial_{j} F \partial_{i}-\partial_{i} F \partial_{j}\right)
$$

with $P_{i, j} \in k\left[t_{0}, \ldots, t_{n}\right]$ homogeneous of equal degrees. Conversely, the theorem below holds.

Theorem 7. Let $V \subseteq \mathbf{P}_{k}^{n}$ be the hypersurface cut out by $F \in k\left[t_{0}, \ldots, t_{n}\right]$ homogeneous of degree $d$. If $V$ is smooth and $p \nmid d$, then each vector field on $\mathbf{P}_{k}^{n}$ leaving $V$ invariant is induced by (4) for certain homogeneous $P_{i, j} \in k\left[t_{0}, \ldots, t_{n}\right]$ of equal degrees.

Proof. The proof we present below is well-known. The argument appeared in [L], Part c of Example 7, p. 892, where it was attributed to Zariski.

Let $X$ be a vector field on $\mathbf{P}_{k}^{n}$ that leaves $V$ invariant, say induced by $\sum G_{i} \partial_{i}$. Then (3) holds for a certain $P \in k\left[t_{0}, \ldots, t_{n}\right]$. Since $p \nmid d$, Euler's formula,

$$
d F=t_{0} \partial_{0} F+\cdots+t_{n} \partial_{n} F
$$

implies that (3) is equivalent to

$$
G_{0}^{\prime} \partial_{0} F+\cdots+G_{n}^{\prime} \partial_{n} F=0
$$

where $G_{i}^{\prime}:=G_{i}-P t_{i} / d$ for $i=0, \ldots, n$. Note that $X$ is also induced by $\sum G_{i}^{\prime} \partial_{i}$.

Let $S:=k\left[t_{0}, \ldots, t_{n}\right]$. Let $I \subseteq S$ be the ideal generated by the partial derivatives of $F$ :

$$
I:=\left(\partial_{0} F, \ldots, \partial_{n} F\right) \subseteq S .
$$

As $p \nmid d$, by Euler's formula, $F \in I$. Since $V$ is smooth, $\sqrt{I}=\left(t_{0}, \ldots, t_{n}\right)$. Hence, the partial derivatives $\partial_{0} F, \ldots, \partial_{n} F$ form a system of parameters for the local ring $\widetilde{S}$, where $\widetilde{S}$ is the localization of $S$ at $\left(t_{0}, \ldots, t_{n}\right)$. Now, since $\widetilde{S}$ is regular, it is Cohen-Macaulay by $[\mathrm{Ma}]$, Thm. 17.8, p. 137. So, the sequence $\partial_{0} F, \ldots, \partial_{n} F$ is regular on $\widetilde{S}$ by $[\mathrm{Ma}]$, Thm. 17.4 , p. 135 . As the polynomials $\partial_{i} F$ are homogeneous, $\partial_{0} F, \ldots, \partial_{n} F$ is regular on $S$ as well, whence its Koszul complex is an exact sequence by [Ma], Thm. 16.5, p. 128. In particular, the module of relations,

$$
R:=\left\{\left(P_{0}, \ldots, P_{n}\right) \in S^{n+1} \mid P_{0} \partial_{0} F+\cdots+P_{n} \partial_{n} F=0\right\},
$$

is generated by the trivial ones,

$$
\partial_{i} F e_{j}-\partial_{j} F e_{i} \quad \text { for } 0 \leq i, j \leq n .
$$


As $\left(G_{0}^{\prime}, \ldots, G_{n}^{\prime}\right) \in R$ by $(5)$, and $X$ is induced by $\sum G_{i}^{\prime} \partial_{i}$, the proof is finished.

Theorem 8. Let $X$ be a non-zero vector field on $\mathbf{P}_{k}^{n}$ of degree $m$. Let $V \subseteq \mathbf{P}_{k}^{n}$ be a hypersurface of degree $d$. If $V$ is smooth, left invariant by $X$, and $p X d$, then $d \leq m+1$.

Proof. By Theorem 7, the vector field $X$ is induced by (4) for certain homogeneous $P_{i, j} \in k\left[t_{0}, \ldots, t_{n}\right]$ of the appropriate degrees. Since $X \neq 0$, we have $P_{i, j} \neq 0$ for certain $i, j$. Since $X$ has degree $m$, we have $\operatorname{deg} P_{i, j}=m-d+1$. As $\operatorname{deg} P_{i, j} \geq 0$, we get $d \leq m+1$.

Remark 9. In the proof of Theorem 7 we observed the following fact: If $V \subseteq \mathbf{P}_{k}^{n}$ is the hypersurface cut out by a homogeneous $F \in k\left[t_{0}, \ldots, t_{n}\right]$ of degree $d$, and $p \nmid d$, then any vector field on $\mathbf{P}_{k}^{n}$ leaving $V$ invariant is induced by $\sum G_{i} \partial_{i}$ for $\left(G_{0}, \ldots, G_{n}\right) \in R$, where $R$ is the module of relations of the partial derivatives of $F$, defined in (6). So, if $q$ is the minimum degree of a non-zero relation in $R$, then the minimum degree of a non-zero vector field on $\mathbf{P}_{k}^{n}$ leaving $V$ invariant is $q$ as well. The number $q$ measures how singular $V$ is. We have $q \leq d-1$ with equality if $V$ is smooth, as seen in the proof of Theorem 7. On the other hand, the worst case $q=0$ occurs if (and only if for $p=0$ ) the hypersurface $V$ is a cone. In $\mathbf{P}_{k}^{2}$ a cone is integral only if it is a line. The plane curve cut out by $F:=t_{0}^{a} t_{1}^{b}-t_{2}^{a+b}$, for $a$ and $b$ coprime, is an example of an integral plane curve with $q=1$. These well-known examples show that the answer to Poincaré's question is negative in general.

10. The dualizing sheaf. If $C \subseteq \mathbf{P}_{k}^{n}$ is a reduced curve with at most ordinary nodes for singularities, then $C$ is a local complete intersection. (Indeed, the completion of the local ring at a point of $C$ is either isomorphic to $k[[t]]$ or $k[[s, t]] /(s t)$, whence the local ring is complete intersection by [Ma], Thm. 21.2, p. 171.) So, the dualizing sheaf of $C$ is invertible by [H], Thm. 7.11, p. 245.

Proposition 11. Let $C \subseteq \mathbf{P}_{k}^{n}$ be a reduced curve with at most ordinary nodes for singularities. Let $\omega$ be the dualizing sheaf of $C$. Let $\mathcal{L}$ be an invertible sheaf on $C$ and $X: \Omega_{C}^{1} \rightarrow \mathcal{L}$ a vector field. If $\omega \otimes \mathcal{L}^{-1}$ is ample, then $X=0$.

Proof. Let $\pi: \widetilde{C} \rightarrow C$ be the normalization of $C$ and $\eta: \Omega_{C}^{1} \rightarrow \pi_{*} \Omega_{\tilde{C}}^{1}$ the adjoint to the induced pullback map. Since $C$ has at most ordinary nodes for singularities, we can check locally that $\eta$ is surjective. As $\pi$ is birational, the kernel of $\eta$ is the torsion sheaf of $\Omega_{C}^{1}$. So

$$
\operatorname{Hom}\left(\Omega_{C}^{1}, \mathcal{L}\right)=\operatorname{Hom}\left(\pi_{*} \Omega_{\tilde{C}}^{1}, \mathcal{L}\right) .
$$


By Serre duality $([\mathrm{H}]$, Ch. III, $\S 7)$ on $C$, the projection formula, and Serre duality on $\widetilde{C}$,

$$
\begin{aligned}
\operatorname{Hom}\left(\pi_{*} \Omega_{\tilde{C}}^{1}, \mathcal{L}\right) & =H^{1}\left(\pi_{*} \Omega_{\tilde{C}}^{1} \otimes \omega \otimes \mathcal{L}^{-1}\right)^{*} \\
& =H^{1}\left(\Omega_{\tilde{C}}^{1} \otimes \pi^{*}\left(\omega \otimes \mathcal{L}^{-1}\right)\right)^{*} \\
& =H^{0}\left(\pi^{*}\left(\mathcal{L} \otimes \omega^{-1}\right)\right) .
\end{aligned}
$$

Now, as $\omega \otimes \mathcal{L}^{-1}$ is ample on $C$, the degree of $\pi^{*}\left(\mathcal{L} \otimes \omega^{-1}\right)$ on each component of $\widetilde{C}$ is negative. Thus $H^{0}\left(\pi^{*}\left(\mathcal{L} \otimes \omega^{-1}\right)\right)=0$, and so $X=0$.

Proposition 12. Let $C \subseteq \mathbf{P}_{k}^{n}$ be a reduced curve with at most ordinary nodes for singularities. Assume $C$ is the complete intersection of hypersurfaces of degrees $d_{1}, d_{2}, \ldots, d_{n-1}$. Let $m$ be an integer and $X: \Omega_{C}^{1} \rightarrow \mathcal{O}_{C}(m-1)$ a vector field. If $m<d_{1}+\cdots+d_{n-1}-n$ then $X=0$.

Proof. The normal bundle of $C$ in $\mathbf{P}_{k}^{n}$ is $\mathcal{O}_{C}\left(d_{1}\right) \oplus \cdots \oplus \mathcal{O}_{C}\left(d_{n-1}\right)$. By $[\mathrm{H}]$, Thm. 7.11, p. 245, the dualizing sheaf $\omega$ of $C$ satisfies

$$
\omega \cong \mathcal{O}_{C}\left(d_{1}+\cdots+d_{n-1}-n-1\right) .
$$

Since $m<d_{1}+\cdots+d_{n-1}-n$, the sheaf $\omega \otimes \mathcal{O}_{C}(1-m)$ is ample, and hence $X=0$ by Proposition 11 .

Theorem 13. Let $X$ be a non-zero vector field on $\mathbf{P}_{k}^{2}$ of degree $m$. Let $C \subseteq \mathbf{P}_{k}^{2}$ be an integral curve of degree $d$ whose only singularities are ordinary nodes. If $C$ is left invariant by $X$ and $p \nmid d$, then $d \leq m+1$.

Proof. The theorem was proved by Cerveau and Lins Neto for $k=\mathbf{C}$; see [CL], Thm. 1 and the remark thereafter on p. 891. We will prove the more general statement above in a forthcoming work. In the next remark, we will show what happens when $p \mid d$.

Remark 14. Let $C \subseteq \mathbf{P}_{k}^{2}$ be the curve cut out by a homogeneous $F \in$ $k\left[t_{0}, \ldots, t_{n}\right]$ of degree $d$ divisible by $p$. Then $C$ is left invariant by a nonzero vector field $X$ on $\mathbf{P}_{k}^{2}$ of degree $d-2$. Moreover, $X$ is unique because $\operatorname{Hom}\left(\Omega_{\mathbf{P}_{k}^{2}}^{1}, \mathcal{O}_{\mathbf{P}_{k}^{2}}(-3)\right)=0$. In concrete terms, write

$$
F=t_{0} H_{0}+t_{1} H_{1}+t_{2} H_{2} \text {. }
$$

Let

$$
G_{0}:=\partial_{1} H_{2}-\partial_{2} H_{1}, \quad G_{1}:=\partial_{2} H_{0}-\partial_{0} H_{2}, \quad G_{2}:=\partial_{0} H_{1}-\partial_{1} H_{0} .
$$

Then

$$
t_{1} G_{2}-t_{2} G_{1}=\partial_{0} F-d H_{0}=\partial_{0} F
$$

where the last equality holds because $p \mid d$. Analogously, $\partial_{1} F=t_{2} G_{0}-t_{0} G_{2}$ and $\partial_{2} F=t_{0} G_{1}-t_{1} G_{0}$. It follows that the vector field induced by $\sum G_{i} \partial_{i}$ leaves $C$ invariant. 
For a numerical example, if $F=t_{2}^{d-1} t_{0}+t_{0}^{d-1} t_{1}+t_{1}^{d-1} t_{2}$ then $X$ is induced by

$$
t_{1}^{d-2} \partial_{0}+t_{2}^{d-2} \partial_{1}+t_{0}^{d-2} \partial_{2}
$$

If $p=0$, the vector field induced by (7) is known as Jouanolou's vector field, and its most striking property is that it leaves no curve invariant for $d \geq 4$; see $[\mathrm{J}]$.

\section{Regularity and central projections}

15. Regularity. Let $\mathcal{F}$ be a coherent sheaf on $\mathbf{P}_{k}^{n}$. Given an integer $m$, we say that $\mathcal{F}$ is $m$-regular if $H^{i}(\mathcal{F}(m-i))=0$ for each positive integer $i$. By $[\mathrm{H}]$, Thm. 5.2, p. 228, there is an integer $m$ such that $\mathcal{F}$ is $m$-regular. If $r$ is minimum among the integers $m$ such that $\mathcal{F}$ is $m$-regular, we call $r$ the Castelnuovo-Mumford regularity, or simply regularity, of $\mathcal{F}$.

Suppose $\mathcal{F}$ is $m$-regular. By the proposition on page 99 of [Mu1], the sheaf $\mathcal{F}(m)$ is generated by its global sections. So, if $\mathcal{F}$ is a non-zero sheaf of ideals, then $m \geq 0$, with equality only if $\mathcal{F}=\mathcal{O}_{\mathbf{P}_{k}^{n}}$. Hence, the regularity of a non-zero coherent sheaf of ideals is well-defined and non-negative. The regularity of $\mathbf{P}_{k}^{n}$ is by definition 1, and the regularity of a proper, closed subscheme of $\mathbf{P}_{k}^{n}$ is that of its sheaf of ideals.

There is also an algebraic notion of regularity, given as follows; see [E], p. 505. Let $S:=k\left[t_{0}, \ldots, t_{n}\right]$ and $M$ a non-zero, finitely generated graded $S$-module. By Hilbert Syzygy Theorem ([E], Cor. 19.7, p. 474), there is a minimal graded free resolution of $M$,

$$
0 \rightarrow F_{m} \rightarrow \cdots \rightarrow F_{i} \rightarrow \cdots \rightarrow F_{0} \rightarrow M \rightarrow 0
$$

of length $m \leq n+1$. For each $i=0, \ldots, m$, let $b_{i}$ be the maximum of the degrees of the generators of $F_{i}$. Let $r:=\max \left(b_{i}-i \mid i=0, \ldots, m\right)$. We say that $r$ is the regularity of $M$. It's clear from the definition that if $J \subset S$ is a non-zero, proper homogeneous ideal with regularity $r$, then the regularity of $S / J$ is $r-1$. By convention, the regularity of the zero module is 1 .

For the correspondence between the algebraic and geometric notions of regularity, see [E], Ex. 20.20, p. 516. In particular, if $V \subseteq \mathbf{P}_{k}^{n}$ is the closed subscheme cut out by a saturated, homogeneous ideal $J \subseteq S$, then the regularity of $V$ is equal to that of $J$. It follows that the regularity of $V$ is an upper bound for the degrees of the hypersurfaces necessary to cut out $V$.

It is a conjecture by D. Eisenbud and S. Goto that the regularity of a nondegenerate, integral closed subscheme $V \subseteq \mathbf{P}_{k}^{n}$ of degree $d$ and codimension $c$ is bounded above by $d-c+1$; see $[\mathrm{EG}], \mathrm{p}$. 93. When $V$ is a curve, this bound was proved by L. Gruson, R. Lazarsfeld and C. Peskine; see [GLP], Thm. 1.1, p. 494. In its full generality, the conjecture has not been proved yet.

Let $V \subseteq \mathbf{P}_{k}^{n}$ be a closed subscheme of pure codimension $c$, and $I \subseteq S$ its saturated, homogeneous ideal. We say that $V$ is arithmetically CohenMacaulay if $S / I$ is Cohen-Macaulay. If $V$ is non-degenerate, arithmetically 
Cohen-Macaulay, integral, and of degree $d$, its regularity is bounded above by $\lceil(d-1) / c\rceil+1$, which is sharper than the bound conjectured by Eisenbud and Goto; see $[\mathrm{T}]$, Cor. 2.3, p. 144.

16. Central projections. Assume $n \geq 2$. Let $S:=k\left[t_{0}, \ldots, t_{n}\right]$. Let $L \subseteq \mathbf{P}_{k}^{n}$ be a linear subspace of dimension $\ell$, with $0 \leq \ell \leq n-2$. Let $s_{0}, \ldots, s_{n-\ell-1} \in S$ be linear forms cutting out $L$. We view $s_{0}, \ldots, s_{n-\ell-1}$ as the homogeneous coordinates of $\mathbf{P}_{k}^{n-\ell-1}$. Let $\bar{S}:=k\left[s_{0}, \ldots, s_{n-\ell-1}\right]$.

Let $V \subseteq \mathbf{P}_{k}^{n}$ be the closed subscheme cut out by a saturated, homogeneous ideal $I \subseteq S$. Assume $L$ does not meet $V$. Then the projection with center $L$ gives rise to a finite map $V \rightarrow \mathbf{P}_{k}^{n-\ell-1}$, whose image $\bar{V} \subseteq \mathbf{P}_{k}^{n-\ell-1}$ is the closed subscheme cut out by the ideal $\bar{I}:=I \cap \bar{S}$. We call $\bar{V}$ the projection of $V$ from the center $L$.

Let $X$ be a vector field on $\mathbf{P}_{k}^{n}$ induced by $\sum G_{i} \partial_{i}$. After a change of coordinates, we may assume $s_{i}=t_{i}$ for $i=0, \ldots, n-\ell-1$. Suppose $X$ leaves $V$ invariant. Then

$$
G_{0} \partial_{0} F+\cdots+G_{n-\ell-1} \partial_{n-\ell-1} F \in I
$$

for each $F \in \bar{I}$. Now, suppose there are $B \in S$ and $\bar{G}_{0}, \ldots, \bar{G}_{n-\ell-1} \in \bar{S}$ homogeneous such that $B G_{i} \equiv \bar{G}_{i} \bmod I$ for each $i=0, \ldots, n-\ell-1$. Then, for each $F \in \bar{I}$, it follows from (8) that

$$
\bar{G}_{0} \partial_{0} F+\cdots+\bar{G}_{n-\ell-1} \partial_{n-\ell-1} F \in \bar{I} .
$$

So the projection $\bar{V}$ is left invariant by the vector field $\bar{X}$ on $\mathbf{P}_{k}^{n-\ell-1}$ induced by $\bar{G}_{0} \partial_{0}+\cdots+\bar{G}_{n-\ell-1} \partial_{n-\ell-1}$. If $X$ is of degree $m$, then $\bar{X}$ is of degree $m+\operatorname{deg} B$. If $V$ is reduced and $L$ is general, then the degree of $\bar{V}$ is equal to that of $V$.

\section{The main theorem}

Theorem 17. Assume $n \geq 2$. Let $\ell$ be an integer such that $0 \leq \ell \leq n-2$. Let $W \subseteq \mathbf{P}_{k}^{n}$ be an arithmetically Cohen-Macaulay closed subscheme of degree $e$, Castelnuovo-Mumford regularity $r$ and pure codimension $\ell+2$. Assume

$$
\widetilde{W}:=\left\{P \in W \mid \text { the tangent space } T_{W, P} \text { has codimension at most } \ell \text { in } \mathbf{P}_{k}^{n}\right\}
$$

has codimension one in $W$. Let $V \subseteq W$ be a reduced, closed subscheme, and $X$ a vector field of degree $m$ on $\mathbf{P}_{k}^{n}$ leaving $V$ invariant. Assume each irreducible component of $V$ contains a non-singular point of $X$. Then, for each general linear subspace $L \subseteq \mathbf{P}_{k}^{n}$ of dimension $\ell$, there is a non-zero vector field of degree $m+e-r$ on $\mathbf{P}_{k}^{n-\ell-1}$ leaving the projection of $V$ from the center $L$ invariant.

Proof. Let $L$ and $L^{\prime}$ be linear subspaces of $\mathbf{P}_{k}^{n}$ of dimensions $\ell$ and $\ell+1$ satisfying $L \subseteq L^{\prime}$. Assume $L$ and $L^{\prime}$ are in general position. We claim that the following three conditions hold:

1. The intersection $W \cap L^{\prime}$ is empty.

2. The projection of $W$ from the center $L$ has degree $e$. 
3. For each irreducible component $C \subseteq V$, the linear subspace of $\mathbf{P}_{k}^{n}$ spanned by $L$ and a general $P \in C$ does not contain the direction given by $X$ at $P$. In fact, since $W$ has pure codimension $\ell+2$, a general linear subspace $L^{\prime} \subseteq \mathbf{P}_{k}^{n}$ of dimension $\ell+1$ does not meet $W$. Thus, Condition 1 holds.

As for Condition 2, let $L^{\prime \prime} \subseteq \mathbf{P}_{k}^{n}$ be a general linear subspace of dimension $\ell+2$ containing $L^{\prime}$. As $W$ is of pure codimension $\ell+2$, the intersection $L^{\prime \prime} \cap W$ is finite, and contains at least one general point $P$ on each irreducible component $C \subseteq W$. Since $L$ is a general subspace of $L^{\prime \prime}$ of dimension $\ell$, we have $L \cap W=\emptyset$ and, for each irreducible component $C \subseteq W$, the linear subspace of $\mathbf{P}_{k}^{n}$ spanned by $L$ and a general $P \in C$ intersects $W$ only at $P$. Now, as $\widetilde{W}$ has codimension 1 in $W$, and $W$ is of pure dimension, for each irreducible component $C \subseteq W$, the general $P \in C$ satisfies $\operatorname{codim}\left(T_{W, P}, \mathbf{P}_{k}^{n}\right)>\ell$. Thus, since $L$ is general of dimension $\ell$, the linear subspace of $\mathbf{P}_{k}^{n}$ spanned by $L$ and $P$ intersects $T_{W, P}$ only at $P$. So, for each irreducible component $C \subseteq W$ and each general $P \in C$, the linear subspace of $\mathbf{P}_{k}^{n}$ spanned by $L$ and $P$ intersects $W$ only at $P$, and with multiplicity 1 . Projecting $W$ with center $L$, we obtain a finite map which is an isomorphism over a dense open subscheme of the image. Hence, the image has degree equal to that of $W$, that is, degree $e$.

Finally, by hypothesis, for each irreducible component $C \subseteq V$, the general $P \in C$ is non-singular for $X$. Since $\ell \leq n-2$, and $L$ is general of dimension $\ell$, the direction given by $X$ at $P$ does not meet $L$. Our claim is proved.

Let $S:=k\left[t_{0}, \ldots, t_{n}\right]$. After changing coordinates, we may assume $L^{\prime}$ is cut out by $t_{0}, \ldots, t_{n-\ell-2}$, and $L$ is cut out by $t_{n-\ell-1}$ in $L^{\prime}$. As $W \cap L^{\prime}=\emptyset$, there is a linear form in $k\left[t_{0}, \ldots, t_{n-\ell-2}\right]$ which is not identically zero on any irreducible component of $W$. Up to subtracting from $t_{n-\ell-1}$ a certain multiple of this form, we may assume $t_{n-\ell-1}$ is not identically zero on any irreducible component of $W$. Let $\bar{S}:=k\left[t_{0}, \ldots, t_{n-\ell-1}\right]$.

Let $J \subseteq S$ denote the saturated, homogeneous ideal cutting out $W$, and put $J^{\prime}:=J+\left(t_{0}, \ldots, t_{n-\ell-2}\right)$. Let $\mathfrak{m}:=\left(t_{0}, \ldots, t_{n}\right)$. As $t_{0}, \ldots, t_{n-\ell-2}$ cut out the empty set in $W$, we have $\sqrt{J^{\prime}}=\mathfrak{m}$. Then the quotient $S / J^{\prime}$ is a vector space of finite dimension over $k$. By hypothesis, $S / J$ is Cohen-Macaulay of pure dimension $n-\ell-1$. So, $S_{\mathfrak{m}} / J_{\mathfrak{m}}$ is a local, Cohen-Macaulay ring of dimension $n-\ell-1$. As $t_{0}, \ldots, t_{n-\ell-2}$ generate in $S_{\mathfrak{m}} / J_{\mathfrak{m}}$ an ideal of dimension zero, they form a regular sequence by [Ma], Thm. 17.4, p. 135. Now, the multiplicity of $S_{\mathfrak{m}} / J_{\mathfrak{m}}$ is the degree of $W$; see [E], Ex. 12.6, p. 276. Since $t_{0}, \ldots, t_{n-\ell-2}$ form a regular sequence in $S_{\mathfrak{m}} / J_{\mathfrak{m}}$, by [E], Ex. 12.11, p. 279, the multiplicity of $S_{\mathfrak{m}} / J_{\mathfrak{m}}$ is equal to the dimension of $S_{\mathfrak{m}} / J_{\mathfrak{m}}^{\prime}$ over $k$, which is the same as the dimension of $S / J^{\prime}$. So $\operatorname{dim}_{k} S / J^{\prime}=e$.

Let $M_{1}, \ldots, M_{e}$ be monomials in $S$ generating $S / J^{\prime}$ over $k$. We may assume $M_{1}=1$. Let $w_{i}:=\operatorname{deg} M_{i}$ for $i=1, \ldots, e$. By [E], Prop. 20.20, p. 508, the regularity of $S / J^{\prime}$ is equal to that of $S / J$, which is $r-1$. Since $S / J^{\prime}$ has finite length, using [E], Ex. 20.18, p. 516, we get

$$
r=\max \left(w_{i} \mid i=1, \ldots, e\right)+1 .
$$


We may assume $w_{e}=r-1$. As $M_{1}, \ldots, M_{e}$ generate $S / J^{\prime}$, for each $i=1, \ldots, e$ there are homogeneous $A_{i, j} \in k\left[t_{0}, \ldots, t_{n-\ell-2}\right]$ for $j=1, \ldots, e$ such that

$$
t_{n-\ell-1} M_{i} \equiv A_{i, 1} M_{1}+\cdots+A_{i, e} M_{e} \bmod J .
$$

Let $A:=\left(A_{i, j}\right)$ and $D:=\operatorname{det}\left(t_{n-\ell-1}-A\right)$. As $M_{0}=1$, and (10) holds for each $i=1, \ldots, e$, it follows that $D \in J$. Clearly, $D$ is monic in $t_{n-\ell-1}$, hence non-zero. In addition, $D$ is homogeneous of degree $e$, because the degree of $A_{i, j}$ is $1+w_{i}-w_{j}$ for each $i, j \in\{1, \ldots, e\}$. As $D \in J \cap \bar{S}$, the projection $\bar{W}$ of $W$ from the center $L$ is contained in the hypersurface cut out by $D$ in $\mathbf{P}_{k}^{n-\ell-1}$. By Condition 2 above, also $\bar{W}$ has degree $e$. So, $D$ cuts out $\bar{W}$.

If $e=1$, also $r=1$. In this case, let $B:=1$. Then $B$ is non-zero, homogeneous of degree $e-r$, and the following property holds.

$$
\text { For each } G \in S \text { there is } \bar{G} \in \bar{S} \text { such that } B G \equiv \bar{G} \bmod J \text {. }
$$

Suppose now that $e>1$. We claim there is $B \in \bar{S}$ non-zero, homogeneous of degree $e-r$, satisfying (11). Indeed, for each $i=1, \ldots, e$, let $D_{i}$ be the determinant of the matrix obtained from $t_{n-\ell-1}-A$ by removing the $i$-th column and the last row. Put $B:=D_{1}$. For each $i=2, \ldots, e$, let $A^{(i)}$ be the matrix obtained from $A$ by exchanging the last row with the row $R^{(i)}:=\left[R_{1}^{(i)}, \ldots, R_{e}^{(i)}\right]$, where $R_{j}^{(i)}:=0$ for $j \in\{2, \ldots, e\}-\{i\}$ and

$$
\left(R_{1}^{(i)}, R_{i}^{(i)}\right):= \begin{cases}\left(M_{i},-1\right) & \text { if } i<e, \\ \left(t_{n-\ell-1} M_{e}, 0\right) & \text { if } i=e .\end{cases}
$$

By (10), modulo $J$, the matrix $A^{(i)}$ has eigenvalue $t_{n-\ell-1}$, with $\left(M_{1}, \ldots, M_{e-1}, 0\right)$ being one eigenvector if $i<e$ and $\left(M_{1}, \ldots, M_{e-1}, M_{e}\right)$ one if $i=e$. Since $M_{1}=1$, it follows that $\operatorname{det}\left(t_{n-\ell-1}-A^{(i)}\right) \in J$. Expanding $\operatorname{det}\left(t_{n-\ell-1}-A^{(i)}\right)$ by the cofactors of the last row, we get

$$
B M_{i} \pm D_{i} \pm t_{n-\ell-1} D_{e} \in J \quad \text { if } i<e
$$

and $t_{n-\ell-1}\left(B M_{e} \pm D_{e}\right) \in J$ if $i=e$. Now, as $t_{n-\ell-1}$ is not identically zero on any irreducible component of $W$, and $W$ is arithmetically Cohen-Macaulay, $t_{n-\ell-1}$ is regular in $S / J$. So $B M_{e} \pm D_{e} \in J$. As $D_{1}, \ldots, D_{e} \in \bar{S}$, and $M_{1}, \ldots, M_{e}$ generate $S / J^{\prime}$, property (11) holds.

We show now that $B \notin J$, whence $B \neq 0$. Indeed, if $B \in J$, as $B M_{e} \pm D_{e} \in J$, we have $D_{e} \in J$. Then, as (12) holds, $D_{i} \in J$ for each $i=2, \ldots, e-1$ as well. Since $D \neq 0$, there is $i \in\{1, \ldots, e\}$ such that $D_{i} \neq 0$. Fix such $i$. As $J \cap \bar{S}$ is generated by $D$, and $D_{i} \in J$, the degree of $D_{i}$ would be at least $e$. However, as $\operatorname{deg} A_{e, i}>0$, the degree of $D_{i}$ is at most $e-1$. We have a contradiction. Hence $B \notin J$. As $w_{e}=r-1$, we have $\operatorname{deg} A_{e, 1}=r$, whence $\operatorname{deg} B=e-r$. So, $B$ is as claimed.

Let $\bar{V}$ be the projection of $V$ from the center $L$. If $B$ cuts out a subscheme containing $\bar{V}$, then $\bar{V}$ is left invariant by the vector field on $\mathbf{P}_{k}^{n-\ell-1}$ induced by $B t_{0}^{m} \partial_{0}$, which is non-zero because $B \neq 0$, and has degree $m+e-r$. The theorem is proved in this case. 
Suppose now that $\bar{V}$ is not contained in the subscheme cut out by $B$. As $V$ is reduced, there is an irreducible component $C \subseteq V$ such that $B(P) \neq 0$ for the general $P \in C$. Let $G_{0}, \ldots, G_{n} \in S$ homogeneous of degree $m$ such that $X$ is induced by $\sum G_{i} \partial_{i}$. By (11), for each $i=0, \ldots, n-\ell-1$, there is $\bar{G}_{i} \in \bar{S}$ such that $B G_{i} \equiv \bar{G}_{i} \bmod J$. Let $\bar{X}$ be the vector field on $\mathbf{P}_{k}^{n-\ell-1}$ induced by $\sum \bar{G}_{i} \partial_{i}$. If $\bar{X} \neq 0$, then $\bar{X}$ has degree $m+e-r$. Moreover, since $X$ leaves $V$ invariant, $\bar{X}$ leaves $\bar{V}$ invariant; see 16 . It remains to show that $\bar{X} \neq 0$. Suppose $\bar{X}=0$. Then there is $H \in \bar{S}$ such that $\bar{G}_{i}=H t_{i}$, whence $B G_{i} \equiv H t_{i}$ $\bmod J$, for each $i=0, \ldots, n-\ell-1$. For the general $P \in C$ we have $B(P) \neq 0$; hence $G_{i}(P) t_{j}(P)=G_{j}(P) t_{i}(P)$ for each $i, j \in\{0, \ldots, n-\ell-1\}$. So, the linear subspace of $\mathbf{P}_{k}^{n}$ spanned by $L$ and $P$ contains the direction given by $X$ at $P$. We get a contradiction with Condition 3 . So $\bar{X} \neq 0$.

Proof. (Theorem $1^{*}$ ) Let $e$ be the degree of $V$. Let $\bar{V} \subseteq \mathbf{P}_{\mathbf{C}}^{s+1}$ be the normalcrossings hypersurface obtained from $V$ by projecting with general center. The degree of $\bar{V}$ is $e$ as well. By Theorem 17, the hypersurface $\bar{V}$ is left invariant by a non-zero vector field on $\mathbf{P}_{\mathbf{C}}^{s+1}$ of degree $m+e-r$. Then $e \leq m+e-r+s+1$ by $[\mathrm{BM}]$. So $r \leq m+s+1$.

Theorem 18. Let $C \subseteq \mathbf{P}_{k}^{n}$ be a reduced curve with degree $d$ and at most ordinary nodes for singularities. Assume $C$ is contained in an arithmetically CohenMacaulay curve $C^{\prime}$ of degree e and Castelnuovo-Mumford regularity $r$ such that the tangent space $T_{C^{\prime}, P}$ of $C^{\prime}$ at $P$ has dimension at most 2 for all but finitely many points $P \in C^{\prime}$. If $C$ is left invariant by a vector field $X$ of degree $m$ on $\mathbf{P}_{k}^{n}$, and contains only finitely many singularities of $X$, then

$$
d \leq m+e-r+2,
$$

with equality only if $C$ is reducible or $p \mid d$. In particular, $r \leq m+2$ if $C=C^{\prime}$.

Proof. Suppose first that $n=2$. Then $r=e$. So, the desired inequality follows from Proposition 12 if $C$ is reducible or $p \mid d$, and Theorem 13 otherwise. We may thus assume $n \geq 3$. Let $\bar{C} \subseteq \mathbf{P}_{k}^{2}$ be the projection of $C$ from a general linear subspace $L \subseteq \mathbf{P}_{k}^{n}$ of dimension $n-3$. Since $C$ has at most ordinary nodes for singularities, and $L$ is general, $\bar{C}$ has at most ordinary nodes as well. (The proofs given to $[\mathrm{H}]$, Prop. 3.5, p. 310 and Thm. 3.10, p. 313 apply.) By Theorem 17 with $V=C$ and $W=C^{\prime}$, there is a non-zero vector field on $\mathbf{P}_{k}^{2}$ of degree $m+e-r$ leaving $\bar{C}$ invariant. Now, apply Proposition 12 or Theorem 13 to $\bar{C}$.

Proof. (Theorem 3) Let $C^{\prime}:=Z \cap H$. Since $Z$ is arithmetically Cohen-Macaulay, and $H$ does not contain any irreducible component of $Z$, also $C^{\prime}$ is arithmetically Cohen-Macaulay by [E], Prop. 18.13, p. 455. It follows from [E], Ex. 12.11, p. 278 that the degree of $C^{\prime}$ is $e f$. In addition, the regularity of $C^{\prime}$ is $r+f-1$. (The proof given to [E], Prop. 20.20, p. 508 applies.) Since $Z$ is smooth at all but finitely many points of $C^{\prime}$, the tangent space of $C^{\prime}$ at all but finitely many 
points has dimension at most 2. From Theorem 18, we get

$$
d \leq m+e f-(r+f-1)+2,
$$

with equality only if $C$ is reducible or $p \mid d$.

Remark 19. Let $V \subseteq \mathbf{P}_{k}^{n}$ be a closed subscheme of pure codimension $e$. Then $V$ is contained in the complete intersection $W$ of $e$ hypersurfaces; see Cor. $4^{*}$ on p. 66 of [Mu2]. The projection from a general center $L \subseteq \mathbf{P}_{k}^{n}$ of dimension $e-2$ maps $V$ to a hypersurface of $\mathbf{P}_{k}^{n-e+1}$. We may apply Theorem 17, as long as we verify that the required condition on the tangent spaces at points of $W$ holds.

If $C \subseteq \mathbf{P}_{k}^{n}$ is a reduced curve, it follows from a repeated application of Thm. 7 on p. 787 of $[\mathrm{AK}]$ that $C$ is contained in a complete intersection surface $Z$ which is non-singular but at the singular points of $C$. Choosing a hypersurface $H \subseteq \mathbf{P}_{k}^{n}$ containing $C$ but no component of $Z$, we may apply Theorem 3 .

Example 20. Let $Q$ be a smooth quadric in $\mathbf{P}_{k}^{3}$. Let $C \subseteq Q$ be an integral curve with bi-degree $(a, b)$ and at most ordinary nodes for singularities. Assume $p \nmid(a+b)$. Assume $C$ is left invariant by a vector field $X$ on $\mathbf{P}_{k}^{3}$ of degree $m$, and $C$ contains finitely many singularities of $X$. Applying Theorem 3 , we get $m \geq \min (a, b)$. Equality may be achieved, and there is in general no bound for $\max (a, b)$ in terms of $m$; see the remark below.

Remark 21. Let $X$ be a vector field on $\mathbf{P}_{k}^{n}$ of degree $m$. We might consider the following question, extending Poincaré's: Can we give a bound for the regularity of a curve in $\mathbf{P}_{k}^{n}$ left invariant by $X$ ? As expected, the answer to this question is no. An example is given in [CCF]: if $X$ is induced by $t_{0} t_{2} \partial_{0}+t_{1} t_{3} \partial_{1}$, for each positive integer $d$ the curve $C_{d} \subseteq \mathbf{P}_{k}^{n}$ cut out by $t_{2}-d t_{3}, t_{0} t_{2}^{d-1}-t_{1}^{d}, t_{4}, \ldots, t_{n}$ has regularity (and degree) $d$ and is left invariant by $X$. This example is quite ingeniously a variation of that given in Remark 9 . Note that $X$ does not depend on $d$. Note however that $C_{d}$ is singular for $d \geq 3$. One might ask whether the answer to the question we raised above is yes if one considers only smooth curves. In the next paragraph, we give an example for $n=3$ to show that a bound cannot be found only in terms of $m$. This example may also be extended to one for each $n \geq 4$.

For each integer $d \geq 1$, let $\mathbf{P}_{k}^{1} \rightarrow \mathbf{P}_{k}^{3}$ be the map defined by taking $\left(t_{0}: t_{1}\right)$ to $\left(t_{0}^{d}: t_{0}^{d-1} t_{1}: t_{0} t_{1}^{d-1}: t_{1}^{d}\right)$, and $C_{d}$ its image. Then $C_{d}$ is non-singular, and is cut out in $\mathbf{P}_{k}^{3}$ by

$$
t_{1} t_{2}-t_{0} t_{3}, \quad t_{1}^{d-1}-t_{2} t_{0}^{d-2}, \quad t_{2}^{d-1}-t_{1} t_{3}^{d-2}
$$

Let $X_{d}$ be the vector field of degree 1 on $\mathbf{P}_{k}^{3}$ induced by

$$
d t_{0} \partial_{0}+(d-2) t_{1} \partial_{1}-(d-2) t_{2} \partial_{2}-d t_{3} \partial_{3}
$$

It's easy to check that $X_{d}$ leaves $C_{d}$ invariant for each positive integer $d$. 


\section{References}

[AK] A. Altman and S. Kleiman, Bertini theorems for hypersurface sections containing a subscheme, Comm. Algebra 7 (1979), no. 8, 775-790.

[BM] M. Brunella and L.G. Mendes, Bounding the degree of solutions to Pfaff equations, Publ. Mat. 44 (2000), no. 2, 593-604.

[CC] A. Campillo, M. Carnicer, Proximity inequalities and bounds for the degree of invariant curves by foliations of $\mathbf{P}_{\mathbf{C}}^{2}$, Trans. Amer. Math. Soc. 349 (1997), no. 6, 2211-2228.

$[\mathrm{CCF}]$ A. Campillo, M. Carnicer and J. García de la Fuente, Invariant curves by vector fields on algebraic varieties, J. London Math. Soc. (2) 62 (2000), no. 1, 56-70.

[C] M. Carnicer, The Poincaré problem in the nondicritical case, Ann. of Math. (2) 140 (1994), no. 2, 289-294.

[CL] D. Cerveau and A. Lins Neto, Holomorphic foliations in $\mathbf{P}_{\mathbf{C}}^{2}$ having an invariant algebraic curve, Ann. Inst. Fourier 41 (1991), no. 4, 883-903.

[E] D. Eisenbud, Commutative algebra. With a view toward algebraic geometry, Graduate Texts in Mathematics, 150. Springer-Verlag, New York, 1995.

[EG] D. Eisenbud and S. Goto, Linear free resolutions and minimal multiplicity, J. Algebra 88 (1984), no. 1, 89-133.

[GLP] L. Gruson, R. Lazarsfeld and C. Peskine, On a theorem of Castelnuovo, and the equations defining space curves, Invent. Math. 72 (1983), no. 3, 491-506.

$[\mathrm{H}] \quad$ R. Hartshorne, Algebraic geometry, Graduate Texts in Mathematics, No. 52. SpringerVerlag, New York-Heidelberg, 1977.

[J] J.P. Jouanolou, Équations de Pfaff algébriques, Lecture Notes in Mathematics, 708. Springer, Berlin, 1979.

[L] J. Lipman, Free derivation modules on algebraic varieties, Amer. J. Math. 87 (1965), $874-898$.

[Ma] H. Matsumura, Commutative ring theory, Second edition. Cambridge Studies in Advanced Mathematics, 8. Cambridge University Press, Cambridge, 1989.

[Mu1] D. Mumford, Lectures on curves on an algebraic surface, Annnals of Mathematics Studies, No. 59, Princeton University Press, Princeton, N.J., 1966.

[Mu2] D. Mumford, The red book of varieties and schemes, Lecture Notes in Mathematics, 1358. Springer-Verlag, Berlin, 1988.

[P] H. Poincaré, Sur l'intégration algébrique des équations differentielles du premier ordre et du premier degré, Rendiconti del Circolo Matematico di Palermo 5 (1891), 161-191.

[Se] E. Sernesi, Topics on families of projective schemes, Queen's Papers in Pure and Applied Mathematics, 73. Queen's University, Kingston, ON, 1986.

[So1] M. Soares, The Poincaré problem for hypersurfaces invariant by one-dimensional foliations, Invent. Math. 128 (1997), no. 3, 495-500.

[So2] M. Soares, Projective varieties invariant by one-dimensional foliations, Ann. of Math. (2) 152 (2000), no. 2, 369-382.

[T] R. Treger, On equations defining arithmetically Cohen-Macaulay schemes. I, Math. Ann. 261 (1982), no. 2, 141-153.

Instituto de Matemática Pura e Aplicada, Estrada Dona Castorina 110, 22460-320 Rio de JANEIRo RJ, BRAZiL.

E-mail address: esteves@impa.br 